PROCEEDINGS OF THE

AMERICAN MATHEMATICAL SOCIETY

Volume 132, Number 5, Pages 1449-1451

S 0002-9939(03)07199-5

Article electronically published on October 8, 2003

\title{
A FORMULA FOR THE JOINT LOCAL SPECTRAL RADIUS
}

\author{
E. YU. EMEL'YANOV AND Z. ERCAN \\ (Communicated by Joseph A. Ball)
}

\begin{abstract}
We give a formula for the joint local spectral radius of a bounded subset of bounded linear operators on a Banach space $X$ in terms of the dual of $X$.
\end{abstract}

Let $X$ be a Banach space and $\mathcal{L}(X)$ the algebra of all bounded linear operators in $X$. The joint spectral radius $\rho(M)$ of a bounded subset $M$ of $\mathcal{L}(X)$ was introduced by G.-C. Rota and W. G. Strang [5] as

$$
\rho(M)=\limsup _{n \rightarrow \infty}\left\|M^{n}\right\|^{1 / n},
$$

where $M^{n}$ is the set of all products $T_{1} \circ \ldots \circ T_{n}\left(T_{i} \in M\right)$ and $\left\|M^{n}\right\|=\sup _{T \in M^{n}}\|T\|$. Recently the notion of the joint local spectral radius $\rho_{x}(M)$ at a point $x \in X$ was introduced by R. Drnovšek [2] for a finite subset $M$ of $\mathcal{L}(X)$ and by V. S. Shulman and Yu. V. Turovskii [6] for a bounded $M \subseteq \mathcal{L}(X)$ as

$$
\rho_{x}(M)=\limsup _{n \rightarrow \infty}\left\|M^{n} x\right\|^{1 / n},
$$

where $\left\|M^{n} x\right\|=\sup _{T \in M^{n}}\|T x\|$. In this note we present the following formula for the joint local spectral radius.

Theorem 1. For any bounded $M \subseteq \mathcal{L}(X)$ and for any $x \in X$ the following holds:

$$
\rho_{x}(M)=\sup _{f \in X^{*}} \limsup _{n \rightarrow \infty}\left|f \circ M^{n}(x)\right|^{1 / n},
$$

where $\left|f \circ M^{n}(x)\right|=\sup \left\{|f \circ T(x)|: T \in M^{n}\right\}$. In particular, $\rho_{x}(M)=0$ if and only if $\lim _{n \rightarrow \infty}\left|f \circ M^{n}(x)\right|^{1 / n}=0$ for all $f \in X^{*}$.

The proof of the theorem is based on a lemma which generalizes the recent result [3, Thm. 3] of S. Onal and the second author in the following way.

Lemma 2. Let $X$ be a Banach space. Then for any sequence $\left(x_{n}\right)_{n}$ in $X$ and for any nonnegative sequence $\left(\epsilon_{n}\right)_{n}$ with $\epsilon_{n} \rightarrow 0$ the following holds:

$$
\limsup _{n \rightarrow \infty}\left\|x_{n}\right\|^{\epsilon_{n}}=\sup _{f \in X^{*}} \limsup _{n \rightarrow \infty}\left|f\left(x_{n}\right)\right|^{\epsilon_{n}} .
$$

Received by the editors November 23, 2002 and, in revised form, January 9, 2003.

2000 Mathematics Subject Classification. Primary 47A11, 47A13, 46H05.

Key words and phrases. Joint local spectral radius, joint spectral radius, Banach algebra.

The work of the first author was supported by the Scientific and Technical Research Council of Turkey. 
Proof. We may assume that the sequence $\left(\epsilon_{n}\right)_{n}$ is decreasing. Denote by $l$ and $r$ the left and the right sides of the formula. Obviously,

$$
r=\sup _{f \in X^{*},\|f\| \leq 1} \limsup _{n \rightarrow \infty}\left|f\left(x_{n}\right)\right|^{\epsilon_{n}} .
$$

Thus $l \geq r$ is trivial, because $\left\|x_{n}\right\| \geq\|f\|\left\|x_{n}\right\| \geq\left|f\left(x_{n}\right)\right|$ for all $f \in X^{*},\|f\| \leq 1$.

Suppose $l>r$. There exists an $\alpha>r$ and a subsequence $\left(x_{n_{k}}\right)_{k}$ of $\left(x_{n}\right)_{n}$ such that $k \epsilon_{n_{k}} \rightarrow 0$ and

$$
\left\|x_{n_{k}}\right\|^{\epsilon_{n_{k}}} \geq \alpha
$$

for all $k$. Put $y_{k}=\alpha^{-\epsilon_{n_{k}}^{-1}} x_{n_{k}}$. Note that $\left\|y_{n_{k}}\right\| \geq 1$ for all $k$ and

$$
\left|f\left(y_{k}\right)\right|=\left|f\left(x_{n_{k}}\right)\right| / \alpha^{1 / \epsilon_{n_{k}}} \rightarrow 0 \quad\left(\forall f \in X^{*}\right),
$$

since

$$
\limsup _{k \rightarrow \infty}\left|f\left(x_{n_{k}}\right)\right|^{\epsilon_{n_{k}}} \leq r<\alpha \quad\left(\forall f \in X^{*}\right) .
$$

Applying the Bessaga-Pelczynski selection principle (see, for example, [1, p. 42]), take a subsequence $\left(y_{k_{i}}\right)_{i}$ that is a basis in the closure of the linear span $Y$ of $\left\{y_{k_{i}}\right\}_{i=1}^{\infty}$. Take the linear functionals $u_{i}$ on $Y$ satisfying

$$
u_{i}\left(y_{k_{j}}\right)=\delta_{i j}, \quad(\forall i, j) .
$$

Then the sequence $\left(\left\|u_{i}\right\|\right)_{i=1}^{\infty}$ is bounded by the basis constant of $\left(y_{k_{i}}\right)_{i}$. Set

$$
u:=\sum_{k=1}^{\infty} 2^{-k} u_{k}
$$

and extend $u$ to a functional $\hat{u} \in X^{*}$. Then $\left|\hat{u}\left(y_{k_{i}}\right)\right|=2^{-i}$ and $\left|\hat{u}\left(x_{n_{k_{i}}}\right)\right|=$ $\alpha^{1 / \epsilon_{n_{k_{i}}}}\left|\hat{u}\left(y_{k_{i}}\right)\right|=2^{-i} \alpha^{1 / \epsilon_{n_{k_{i}}}}$ for each $i$. On the other hand,

$$
\limsup _{i \rightarrow \infty}\left|\hat{u}\left(x_{n_{k_{i}}}\right)\right|^{\epsilon_{n_{k_{i}}}} \leq r
$$

implies that

$$
\limsup _{i \rightarrow \infty} 2^{-i \epsilon_{n_{k_{i}}}} \leq r / \alpha<1,
$$

which contradicts $i \epsilon_{n_{k_{i}}} \rightarrow 0$.

Proof of Theorem [1. Denote by $l$ and $r$ the left and the right sides of (1). The inequality $l \geq r$ is trivial. Suppose that $l>r$. Then there exists a sequence $\left(T_{n_{k}} \in M^{n_{k}}\right)_{k}$ such that

$$
\left\|T_{n_{k}} x\right\|^{1 / n_{k}} \geq \alpha>r
$$

for some $\alpha$ and for all $k$. Applying Lemma 2 to the sequences $\left(x_{k}\right)_{k}, x_{k}=T_{n_{k}} x$ and $\left(\epsilon_{k}\right)_{k}, \epsilon_{k}=1 / n_{k}$ we find an $f \in X^{*}$ such that

$$
\limsup _{k \rightarrow \infty}\left|f \circ T_{n_{k}}(x)\right|^{1 / n_{k}}>r
$$

which contradicts

$$
r=\sup _{f \in X^{*}} \limsup _{n \rightarrow \infty}\left|f \circ M^{n}(x)\right|^{1 / n} .
$$

Consequently, $l=r$ and the proof of the theorem is complete. 
As an application of Theorem 1 we give the following formula for the joint spectral radius of bounded subsets of a Banach algebra. As usual, for a bounded subset $M$ of a Banach algebra $A$, by $\rho(M)$ is denoted the joint spectral radius $\rho(M)=\lim \sup _{n \rightarrow \infty}\left\|M^{n}\right\|^{1 / n}$ of $M$ (see, for example, [4]). The relation between the joint spectral radius and the geometric spectral radius of noncommuting Banach algebra elements is investigated by P. Rosenthal and A. Soltysiak in [4].

Corollary 3. Let $A$ be a Banach algebra and $M$ a bounded subset of $A$. Then

$$
\rho(M)=\sup _{f \in A^{*}} \limsup _{n \rightarrow \infty}\left|f\left(M^{n}\right)\right|^{1 / n},
$$

where $\left|f\left(M^{n}\right)\right|=\sup \left\{|f(a)|: a \in M^{n}\right\}$. In particular, $\rho(M)=0$ if and only if $\lim _{n \rightarrow \infty}\left|f\left(M^{n}\right)\right|^{1 / n}=0$ for all $f \in A^{*}$.

Proof. We may assume that the algebra $A$ has a unit, say $e$. Consider the bounded subset $\mathcal{M}=\left\{T_{a}: a \in M\right\}$ of $\mathcal{L}(A)$, where $T_{a}$ is defined as the left multiplication $T_{a}(x)=a x$. Then $\rho(M)=\rho_{e}(\mathcal{M})$ and $\left|f\left(M^{n}\right)\right|=\left|f \circ \mathcal{M}^{n}(e)\right|$ for any $f$ and $n$. To complete the proof it is enough to apply Theorem 1 to $\mathcal{M} \subseteq \mathcal{L}(A)$ and $e$.

\section{REFERENCES}

[1] J. Diestel, Sequences and series in Banach spaces, Graduate Texts in Mathematics 92, Springer-Verlag, New York (1984). MR 85i:46020

[2] R. Drnovšek, On reducibility of semigroups of compact quasinilpotent operators, Proc. Amer. Math. Soc. 125 (1997), 2391-2394. MR 97m:47007

[3] Z. Ercan and S. Onal, Invariant subspaces for positive operators acting on a Banach space with Markuhevich basis, Positivity. (to appear)

[4] P. Rosenthal and A. Soltysiak, Formulas for the joint spectral radius of noncommuting Banach algebra elements, Proc. Amer. Math. Soc. 123 (1995), 2705-2708. MR 95k:47008

[5] G.-C. Rota and W. G. Strang, A note on the joint spectral radius, Indag. Math. 22 (1960), 379-381. MR 26:5434

[6] V. S. Shulman and Yu. V. Turovskii, Joint spectral radius, operator semigroups, and a problem of W. Wojtyński, J. Funct. Anal. 177 (2000), 383-441. MR 2002d:47099

Sobolev Institute of Mathematics, AcAd. Koptyug PR. 4, 630090 Novosibirsk, Russia Current address: Department of Mathematics, Middle East Technical University, 06531 Ankara, Turkey

E-mail address: emelanov@math.nsc.ru

Department of Mathematics, Middle East Technical University, 06531 Ankara, TURKEY

E-mail address: zercan@metu.edu.tr 